IP Periodica Polytechnica Civil Engineering

\author{
60(3), pp. $413,420,2016$ \\ DOI: $10.3311 /$ PPci.8198 \\ Creative Commons Attribution (1) \\ RESEARCH ARTICLE
}

\section{Lyapunov Based Control Algorithm for Seismically Excited Buildings}

Farida Achour-Olivier, Hamid Afra

Received 03-05-2015, revised 28-09-2015, accepted 16-01-2016

\begin{abstract}
Several different seismic active control algorithms have been proposed in the last decades, most of these studies are based on the applications of the traditional linear quadratic regulator control (LQR). This paper develops a new control algorithm for SDOF structures based in Lyapunov method. This algorithm uses Lyapunov's direct approach for stability analysis in design of feedback controller. The approach requires the use of Lyapunov function candidate, which must be a positive definite function of the states of the system. The controller is designed so as to make the derivative of the Lyapunov function negative semidefinite. Numerical simulations using one story frame structure modeled as shear building structure subjected to earthquake excitations have been performed to evaluate the effectiveness of the proposed algorithm.
\end{abstract}

\section{Keywords}

Seismic active control · Lyapunov function - Lyapunov stability theory $\cdot$ Numerical simulation

\section{Farida Achour-Olivier}

Faculty of Civil Engineering, University of Science and Techology, Houari Boumediene (USTHB), BP 32 El Alia 16111 Bab Ezzouar, Algiers, Algeria e-mail: achfarida@yahoo.fr, fachour@usthb.dz

\section{Hamid Afra}

National Center of Building Integrated Research and Studies (CNERIB), Cité El Mokrani Souidania, Algiers, Algeria

e-mail: afra_hamid@yahoo.com

\section{Introduction}

The application of modern control techniques to mitigate the effects of seismic loads on civil engineering structures offers an appealing alternative to traditional earthquake resistant design approaches, and enhances safety of structures under extreme conditions, especially for strategic buildings. There has been a large amount of research effort devoted to the theoretical and practical development of structural control, especially in countries where earthquakes are active [1,2]. Structural control can be applied to mitigate the vibration amplitudes by using appropriate devices and control techniques [3]. The key element in successful use of these devices and techniques is an effective control algorithm to compute loads to be applied to the structure, and it demonstrated that the performance of the controlled system is highly dependent on the choice of algorithm [4]. An effective control algorithm has to be robust and functional under various dynamic conditions. Various control algorithms have been proposed, and based in different optimization procedures including linear quadratic regulator (LQR) [5, 6]. The LQR control is among the well-known optimal algorithms mainly due to its simplicity and ease of implementation. Then, it is regarded as a baseline in modern control theory [6]. Even though it can be used to reduce vibrations, it suffers from a number of shortcomings, such as being susceptible to parameters uncertainty and modelling error. Another drawback is the difficulty to study the stability of controlled structures [6]. However, for the practical application of an active control method to civil engineering structures, the problem of stability and robustness is one of the major issues and it has been examined in several studies [7]. Design of a stable and robust controller is possible by using Lyapunov stability theory. This approach requires the definition of Lyapunov function which must be positive definite [7,-9], and the corresponding controller is designed so as to make the derivative of the Lyapunov function negative semi-definite [7,9]. The importance of this approach is the fact that it allows the stability of the system to be controlled without solving the differential equation explicitly. Lyapunov's second method has today become the method of choice in determining the stability of a control design for a dynamical system [9, 10]. 
In general, there is no standard method or systematic procedure to determine an appropriate Lyapunov function that ensures that its derivative is negative semi definite [10]. Therefore, there are a Lyapunov functions in quadratic form often used.

Although Lyapunov's direct method is efficient for stability analysis, it is of restricted applicability due to the difficulty in determining a Lyapunov function

Wu and Soong proposed a modified bang-bang control using Lyapunov's direct method [11]. Min and al. formulated a probabilistic control algorithm, which determines the direction of a control load by the Lyapunov controller design method [7, 12]. Lee and al proposed a modified sliding mode control algorithm using a target derivative of the Lyapunov function [7]. Hiramoto and al. presented an inverse Lyapunov method in bang-bang semi active control for civil engineering structures [13].

In this paper, a Lyapunov based seismic control algorithm is developed for a single degree of freedom (SDOF) civil engineering structures subjected to a seismic ground motion. It is important to note that for its application, this proposed control algorithm needs to know the mass, damping and stiffness of the structure.

The Lyapunov function candidate is a quadratic function of displacement and velocity. The control law is determined so to make the rate of change of the Lyapunov function as negative as possible.

To evaluate the effectiveness of the proposed algorithm, numerical simulations on controlled and uncontrolled seismic behavior of a single story shear building structure have been conducted. The structure analysis and control design are carried out using a computer program developed with MATLAB [14].

\section{Problem formulation and mathematical modeling}

Consider a single degree of freedom (SDOF) structure subjected to a seismic ground excitation $\ddot{x}_{g}(t)$. The equation of motion of this structure is expressed as:

$$
m \ddot{x}+c \dot{x}+k x=-m \ddot{x}_{g}(t)
$$

Where:

$\begin{array}{ll}m & \text { Mass of the structure, } \\ c & \text { Damping of the structure, } \\ k & \text { Stiffness of the structure, } \\ x & \text { Floor displacement } \\ \dot{x} & \text { Floor velocity, } \\ \ddot{x} & \text { Floor acceleration. }\end{array}$

Assuming that a control force $u(t)$ is applied to the structure, the equation of motion becomes:

$$
m \ddot{x}+c \dot{x}+k x=-m \ddot{x}_{g}(t)+u(t)
$$

Using the state space concept, Eq. (2) can be written as:

$$
\dot{Z}(t)=A Z(t)+B_{u} u(t)+B_{r} \ddot{x}_{g}(t)
$$

Where:

$Z(t)$ State vector given by:

$$
Z(t)=\left(\begin{array}{c}
x(t) \\
\dot{x}(t)
\end{array}\right)
$$

$A$ System state matrix given by:

$$
A=\left[\begin{array}{cc}
0 & 1 \\
-\frac{k}{m} & -\frac{c}{m}
\end{array}\right]
$$

$B_{u}$ Control location vector given by:

$$
B_{u}=\left[\begin{array}{c}
0 \\
\frac{1}{m}
\end{array}\right]
$$

$B_{r}$ Excitation application vector given by:

$$
B_{r}=\left[\begin{array}{c}
0 \\
-1
\end{array}\right]
$$

The control force $u(t)$ will be determined using Lyapunov stability theory.

\section{Lyapunov based control design}

In this study, the Lyapunov's direct method is considered for feedback controller design. The idea is that, by first choosing a Lyapunov function candidate and then the feedback control law is determined such that it makes the derivative of the specified Lyapunov function candidate negative semi definite. This way of designing control is called Lyapunov design. Lyapunov design depends on the selection of Lyapunov functions. It's known that an important class of candidate Lyapunov function is that of the quadratic ones [10].

\subsection{Control algorithm}

Let's assume a control law in the form of state feedback as:

$$
u(t)=-G Z(t)=-\left[\begin{array}{ll}
g_{d} & g_{v}
\end{array}\right]\left(\begin{array}{c}
x(t) \\
\dot{x}(t)
\end{array}\right)
$$

Where:

$G \quad$ Control gain matrix, $\left(\begin{array}{l}x(t) \\ \dot{x}(t)\end{array}\right)$ State vector.

When the input to a system depends on that system's output, the resulting closed loop control is referred to as feedback control.

For a closed loop control, Eq. 3 is expressed as:

$$
\dot{Z}(t)=A_{c} Z(t)+B_{r} \ddot{x}_{g}(t)
$$

Where:

$$
A_{c}=A-\left(B_{u} G\right)=\left[\begin{array}{cc}
0 & 1 \\
-\frac{k}{m}-\frac{g_{d}}{m} & -\frac{c}{m}-\frac{g_{v}}{m}
\end{array}\right]
$$


For a controller design, let us consider the Lyapunov function candidate as:

$$
V\left(z_{1}, z_{2}\right)=\left(\frac{\beta^{2}}{4}+\alpha\right) z_{1}^{2}+\left(z_{2}+\frac{\beta z_{1}}{2}\right)^{2}
$$

Where:

$$
\begin{aligned}
& z_{1}=x(t) \\
& z_{2}=\dot{x}(t)
\end{aligned}
$$

For the sake of simplicity, $\alpha$ and $\beta$ are defined as:

$$
\begin{aligned}
& \alpha=\omega^{2}+G_{d} \\
& \beta=2 \xi \omega+G_{v}
\end{aligned}
$$

Where:

$$
\begin{aligned}
\omega^{2} & =\frac{k}{m} \\
2 \xi \omega & =\frac{c}{m} \\
G_{d} & =\frac{g_{d}}{m} \\
G_{v} & =\frac{g_{v}}{m}
\end{aligned}
$$

The function given in (11) can be written in quadratic form as the following:

$$
V=Z^{T} P Z
$$

Where:

$$
P=\left[\begin{array}{cc}
\left(\frac{\beta^{2}}{2}\right)+\alpha & \frac{\beta}{2} \\
\frac{\beta}{2} & 1
\end{array}\right]
$$

For the Lyapunov function candidate given in Eq. (11), its time derivative is defined as:

$$
\dot{V}=\frac{\partial V}{\partial t}=\dot{z}_{1} \frac{\partial V}{\partial z_{1}}+\dot{z}_{2} \frac{\partial V}{\partial z_{2}}
$$

Where:

$$
\begin{gathered}
\frac{\partial V}{\partial z_{1}}=2\left(\frac{\beta^{2}}{4}+\alpha\right) z_{1}+\left(2 z_{2}+\beta z_{1}\right) \frac{\beta}{2} \\
\frac{\partial V}{\partial z_{2}}=2\left(z_{2}+\frac{\beta}{2} z_{1}\right) \\
\dot{z}_{1}=z_{2} \\
\dot{z}_{2}=-\left(\omega^{2}+G_{d}\right) z_{1}-\left(2 \xi \omega+G_{v}\right) z_{2}-a(t) \\
a(t)=\ddot{x}_{g}(t)
\end{gathered}
$$

By substituting Eq. 22, to 26 into Eq. 21, it results $\dot{V}$ as:

$$
\dot{V}=-\beta\left(z_{2}+\frac{a(t)}{\beta}\right)^{2}-\alpha \beta\left(z_{1}+\frac{a(t)}{2 \alpha}\right)^{2}+\frac{a^{2}(t)}{\beta}+\frac{\beta a^{2}(t)}{4 \alpha}
$$

It can be verified that to make a Lyapunov function, given by Eq. (11), positive definite, the parameter $\alpha$ must be positive, $V>0$ If $\alpha>0$. Also, to make its derivative, given by Eq. 27, negative, the parameters $\alpha$ and $\beta$ must be positive, $\dot{V}<0$ If $\alpha, \beta>0$

The control force $u(t)$ is determined based on the parameters $\alpha$ and $\beta$.

\subsection{Gain matrix parameters}

To obtain the control gain matrix $G$, the parameters $\alpha$ and $\beta$ must be determined. For this, one makes the function derivative equal zero, $\dot{V}=0$

$$
\begin{aligned}
& \dot{V}=0 \Rightarrow \\
& \beta\left(z_{2}+\frac{a(t)}{\beta}\right)^{2}+\alpha \beta\left(z_{1}+\frac{a(t)}{2 \alpha}\right)^{2}=\frac{a^{2}(t)}{\beta}+\frac{\beta a^{2}(t)}{4 \alpha}
\end{aligned}
$$

Equation 28 can be replaced by the following inequalities system as:

$$
\begin{gathered}
\left(z_{1}+\frac{a(t)}{2 \alpha}\right)^{2} \leq \frac{1}{\alpha \beta}\left(\frac{a^{2}(t)}{\beta}+\frac{\beta a^{2}(t)}{4 \alpha}\right) \\
\left(z_{2}+\frac{a(t)}{\beta}\right)^{2} \leq \frac{1}{\beta}\left(\frac{a^{2}(t)}{\beta}+\frac{\beta a^{2}(t)}{4 \alpha}\right)
\end{gathered}
$$

After rearrangement, inequality $(29)$ becomes

$$
\begin{gathered}
\left(z_{1}+\frac{a(t)}{2 \alpha}\right)^{2} \leq a^{2}(t)\left(\frac{1}{\alpha \beta^{2}}+\frac{1}{4 \alpha^{2}}\right) \\
-|a(t)| \sqrt{\frac{1}{\alpha \beta^{2}}+\frac{1}{4 \alpha^{2}}} \leq\left(z_{1}+\frac{a(t)}{2 \alpha}\right) \leq|a(t)| \sqrt{\frac{1}{\alpha \beta^{2}}+\frac{1}{4 \alpha^{2}}} \\
-|a(t)| \sqrt{\frac{1}{\alpha \beta^{2}}+\frac{1}{4 \alpha^{2}}}-\frac{a(t)}{2 \alpha} \leq z_{1} \leq|a(t)| \sqrt{\frac{1}{\alpha \beta^{2}}+\frac{1}{4 \alpha^{2}}}-\frac{a(t)}{2 \alpha}
\end{gathered}
$$

For the sake of simplicity, $\mathrm{D}_{1}$ is defined as: $D_{1}=\frac{1}{\alpha \beta^{2}}+\frac{1}{4 \alpha^{2}}$ Consequently, 33 can be rewritten as:

$$
-|a(t)| \sqrt{D_{1}}-\frac{a(t)}{2 \alpha} \leq z_{1} \leq|a(t)| \sqrt{D_{1}}-\frac{a(t)}{2 \alpha}
$$

The ground acceleration can be expressed as

$$
a(t)=|a(t)| \operatorname{sgna}(t)
$$

The sgn in equation (35) stands for sign of $a(t): \operatorname{sgn} a(t)=$ +1 if $a(t)>0$, and $=-1$ if $a(t)<0$

By substituting Eq. (35) into 34, it results

$$
\frac{-|a(t)| \operatorname{sgn} a(t)}{2 \alpha}-|a(t)| \sqrt{D_{1}} \leq z_{1} \leq|a(t)| \sqrt{D_{1}}-\frac{|a(t)| \operatorname{sgna} a(t)}{2 \alpha}
$$




$$
|a(t)|\left(-\frac{\operatorname{sgna}(t)}{2 \alpha}-\sqrt{D_{1}}\right) \leq z_{1} \leq|a(t)|\left(\sqrt{D_{1}}-\frac{\operatorname{sgna}(t)}{2 \alpha}\right)
$$

Consequently, depending on the sign of $a(t)$, 37 can be expressed as:

$$
\begin{aligned}
& a(t)>0 \\
& \Rightarrow|a(t)|\left(-\frac{1}{2 \alpha}-\sqrt{D_{1}}\right) \leq z_{1} \leq|a(t)|\left(\sqrt{D_{1}}-\frac{1}{2 \alpha}\right) \\
& a(t)<0 \\
& \Rightarrow|a(t)|\left(\frac{1}{2 \alpha}-\sqrt{D_{1}}\right) \leq z_{1} \leq|a(t)|\left(\sqrt{D_{1}}+\frac{1}{2 \alpha}\right)
\end{aligned}
$$

Then, inequalities (38) and (39) can lead to one inequality as:

$$
|a(t)|\left(-\frac{1}{2 \alpha}-\sqrt{D_{1}}\right) \leq z_{1} \leq|a(t)|\left(\sqrt{D_{1}}+\frac{1}{2 \alpha}\right)
$$

Following the same procedure and similarly treatment for inequality (30), we obtain

$$
|a(t)|\left(-\frac{1}{\beta}-\sqrt{D_{2}}\right) \leq z_{2} \leq|a(t)|\left(\sqrt{D_{2}}+\frac{1}{\beta}\right)
$$

Where $D_{2}$ is defined as:

$$
D_{2}=\frac{1}{\beta^{2}}+\frac{1}{4 \alpha}
$$

Inequalities (40) and (41) can be rewritten as:

$$
\begin{array}{r}
-|a(t)|\left(+\frac{1}{2 \alpha}+\sqrt{D_{1}}\right) \leq z_{1} \leq|a(t)|\left(\sqrt{D_{1}}+\frac{1}{2 \alpha}\right) \\
-|a(t)|\left(+\frac{1}{\beta}+\sqrt{D_{2}}\right) \leq z_{2} \leq|a(t)|\left(\sqrt{D_{2}}+\frac{1}{\beta}\right)
\end{array}
$$

It can be shown that inequalities (43) and 44 can be expressed by using the peak ground acceleration (PGA)

$a_{\max }$, maximal displacement and maximal velocity of the structure $z_{1 \max }$ and $z_{2 \max }$ respectively as

$$
\left\{\begin{array}{c}
a_{\max }\left(\frac{1}{2 \alpha}+\sqrt{D_{1}}\right)=z_{1 \max } \\
a_{\max }\left(\frac{1}{\beta}+\sqrt{D_{2}}\right)=z_{2 \max }
\end{array}\right\}
$$

The parameters $\alpha$ and $\beta$ will be obtained by equation (45). For this, let's introduce a factor $D$ defined as:

$$
D=\frac{1}{\alpha \beta^{2}}+\frac{1}{4 \alpha^{2}}
$$

Using the factor $D$, the system 45 can be rewritten as:

$$
\left\{\begin{array}{l}
a_{\max }\left(\sqrt{D}+\frac{1}{2 \alpha}\right)=z_{1 \max } \\
a_{\max }\left(\sqrt{\alpha D}+\frac{1}{\beta}\right)=z_{2 \max }
\end{array}\right\}
$$

The system 47) is rearranged in such a manner to have an algebraic equation system with $\alpha$ and $\beta$ as unknowns:

$$
\left\{\begin{array}{c}
a_{\max } z_{1 \max } \beta^{2}-z_{1 \max }^{2} \alpha \beta^{2}+a_{\max }^{2}=0 \\
a_{\max }^{2} \beta-4 z_{2 \max }^{2} \alpha \beta+8 a_{\max } z_{2 \max } \alpha=0
\end{array}\right\}
$$

From the first equation of the system (48), we obtain expression of the parameter $\alpha$ as:

$$
\alpha=\frac{a_{\max }^{2} \beta}{4 z_{2 \max }^{2} \beta-8 a_{\max } z_{2 \max }}
$$

By substituting Eq. (49) into the second equation of the system (48), it results the expression of the parameter $\beta$ as:

$$
a \beta^{3}+b \beta^{2}+c \beta+d=0
$$

Where

$$
\begin{array}{ll}
a & =a_{\max }^{2} z_{1 \max }^{2}-4 z_{2 \max }^{2} z_{1 \max } a_{\max } \\
b & =8 a_{\max }^{2} z_{1 \max } z_{2 \max } \\
c & =-4 a_{\max }^{2} z_{2 \max }^{2} \\
d & =8 a_{\max }^{3} z_{2 \max }
\end{array}
$$

Once the Eq. (50) is resolved, the gain matrix will be determined as a feedback control law.

\section{Numerical example and simulation}

Aldemir and Bakioglu [5] proposed a closed loop control algorithm by forcing the rate of change of the system energy to be negative as possible. This control algorithm has been developed for a single degree of freedom structure and a linear quadratic regulator control is used for results comparison. Motivated by these considerations, the single story frame structure used by Aldemir and Bakioglu [5] is considered to evaluate the effectiveness of the proposed algorithm. The behavior of this structure is analyzed for the cases of uncontrolled, LQR control and this proposed Lyapunov based control.

Let's remind briefly how the linear quadratic regulator (LQR) is applied for the system represented in equation (3).

In this case, the control gain matrix is determined as:

$$
G=-\frac{1}{2} R^{-1} B_{u}^{T} p
$$

Where:

$R \quad$ Weighting matrix for the control force vector,

$p \quad$ Solution of Ricatti equation given as:

$$
A^{T} p+p A-\frac{1}{2} p B_{u} R^{-1} B_{u}^{T} p+2 Q=\underline{\underline{0}}
$$

Where:

$Q \quad$ Weighting matrix for the state vector,

The structure considered is a one story shear building. The structural mass and stiffness have been concentrated in floor and columns respectively. The structural parameters are $m=50$ tons, $k=47000 \mathrm{kN} / \mathrm{m}, c=90 \mathrm{kN} \mathrm{s} / \mathrm{m}$. 
The simulations were performed under three historical earthquake excitations with different frequency content:

- El Centro 1940 accelerogram with peak ground acceleration (PGA) of $3.417 \mathrm{~m} / \mathrm{s}^{2}$.

- Northridge 1994 accelerogram with PGA of $5.926 \mathrm{~m} / \mathrm{s}^{2}$;

- Mexico 1978 accelerogram with PGA of $2.993 \mathrm{~m} / \mathrm{s}^{2}$.

These records have been extensively used in various research studies and practical control applications throughout the world. Their accelerograms are shown in Figure 1.

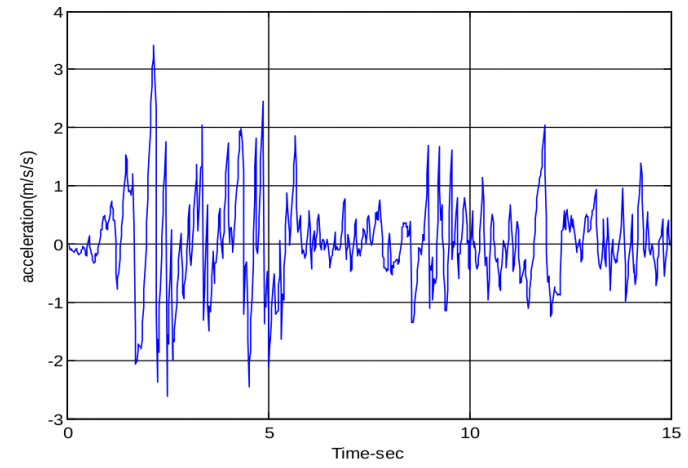

(a) El Centro ground motion

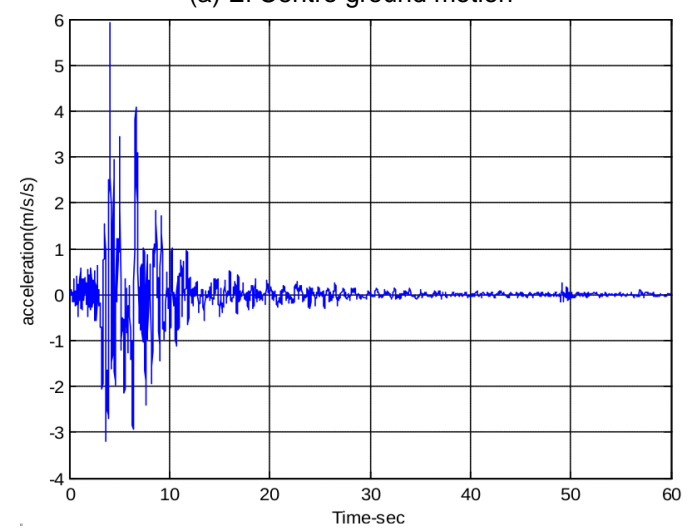

(b) Northridge ground motion

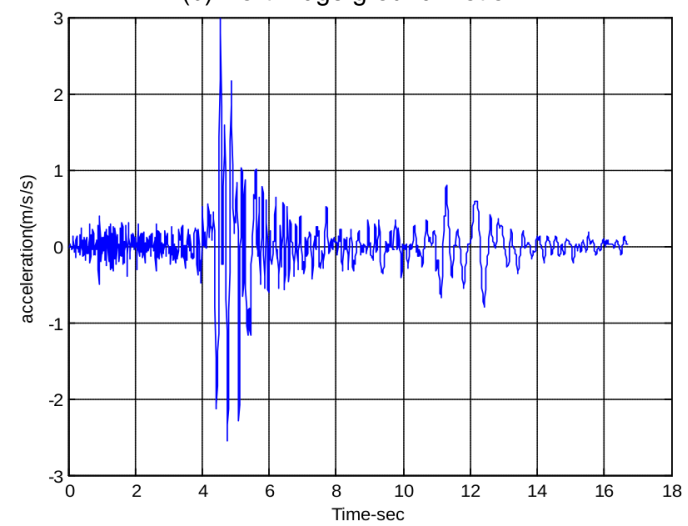

(c) Mexico ground motion

Fig. 1. Excitation accelerograms

To evaluate the control algorithm in terms of reduction of peak responses, the following evaluation criteria adopted from [15, 16] are considered:

1 Story displacement ratio

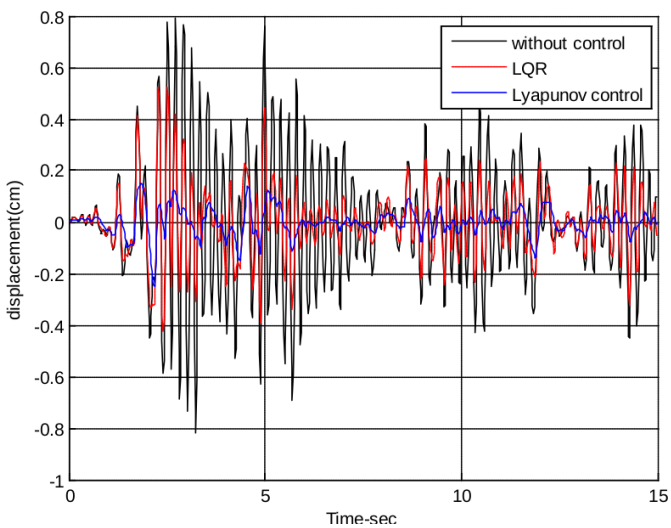

(a) Story displacement

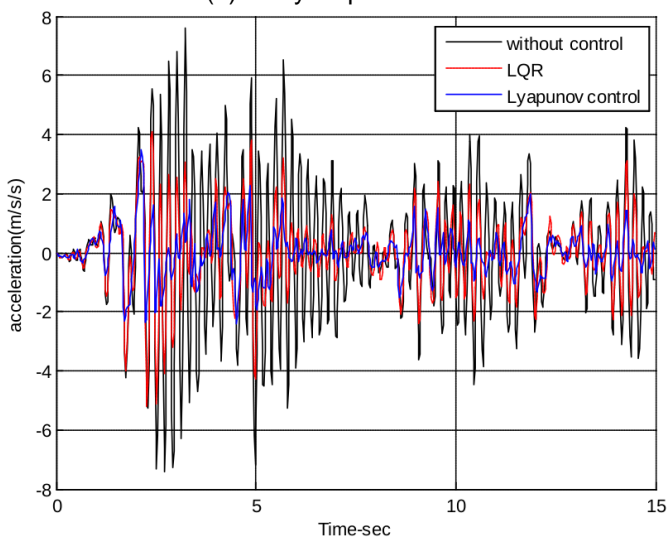

(b) Story acceleration

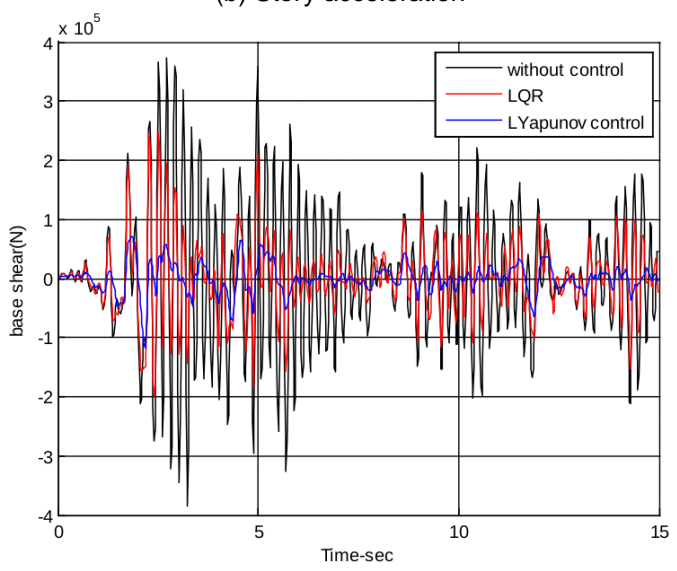

(c) Story base shear

Fig. 2. Uncontrolled and controlled structure responses under El Centro record

$$
J_{1}=\frac{\max \left|y_{c}\right|}{\max \left|y_{u}\right|}
$$

Where

$y_{c}$ Controlled story displacement,

$y_{u}$ Uncontrolled story displacement.

2 Story acceleration ratio

$$
J_{2}=\frac{\max \left|\ddot{y}_{c}\right|}{\max \left|\ddot{y}_{u}\right|}
$$

Where

$\ddot{y}_{c}$ Controlled story acceleration,

$\ddot{y}_{u}$ Uncontrolled story acceleration. 
Tab. 1. Maximum displacement, acceleration and base shear

\begin{tabular}{cccc}
\hline & Displacement $(\mathrm{cm})$ & Acceleration $\left(\mathrm{m} / \mathrm{s}^{2}\right)$ & Base Shear $(\mathrm{kN})$ \\
\hline El-Centro 1940 & & & \\
\hline Uncontrolled & 0.82 & 7.62 & 384.25 \\
LQR control & 0.52 & 5.20 & 245.95 \\
Lyapunov based control & 0.25 & 3.48 & 117.42 \\
\hline Northridge 1994 & & & \\
\hline Uncontrolled & 0.99 & 9.39 & 465.29 \\
LQR control & 0.74 & 7.15 & 346.53 \\
Lyapunov based control & 0.19 & 5.92 & 86.99 \\
\hline Mexico 1978 & & & \\
\hline Uncontrolled & 0.66 & 6.28 & 311.90 \\
LQR control & 0.57 & 5.53 & 61.180 \\
Lyapunov based control & 0.13 & 3.15 & \\
\hline
\end{tabular}

Tab. 2. Responses Ratios (evaluation criteria)

\begin{tabular}{|c|c|c|c|c|}
\hline & J1 & J2 & J3 & $\mathrm{J} 4$ \\
\hline \multicolumn{5}{|l|}{ El-Centro 1940} \\
\hline LQR control & 0.6401 & 0.6830 & 0.5474 & 0.5664 \\
\hline $\begin{array}{l}\text { Lyapunov based } \\
\text { control }\end{array}$ & 0.3056 & 0.4573 & 0.2149 & 0.3482 \\
\hline \multicolumn{5}{|l|}{ Northridge 1994} \\
\hline LQR control & 0.7448 & 0.7616 & 0.7367 & 0.7596 \\
\hline $\begin{array}{l}\text { Lyapunov based } \\
\text { control }\end{array}$ & 0.1870 & 0.6313 & 0.1970 & 0.5341 \\
\hline \multicolumn{5}{|l|}{ Mexico 1978} \\
\hline LQR control & 0.8565 & 0.8810 & 0.7621 & 0.7916 \\
\hline $\begin{array}{l}\text { Lyapunov based } \\
\text { control }\end{array}$ & 0.1962 & 0.5019 & 0.2759 & 0.5040 \\
\hline
\end{tabular}

3 Root mean square (RMS) story displacement ratio

$$
J_{3}=\frac{\vec{y}_{c}}{\vec{y}_{u}}
$$

$\vec{y}_{c}, \vec{y}_{u}$ are calculated from:

$$
\vec{y}=\operatorname{std}(y) * \sqrt{\left(T_{s} / T_{f}\right)}
$$

Where

$T_{s}$ Sampling time,

$T_{f}$ Total excitation duration,

std Standard deviation.

4 Root mean square (RMS) story acceleration ratio

$$
J_{4}=\frac{\vec{y}_{c}}{\vec{y}_{u}}
$$

$\vec{y}_{c}, \vec{y}_{u}$ are calculated from

$$
\vec{y}=\operatorname{std}(\ddot{y}) * \sqrt{\left(T_{s} / T_{f}\right)}
$$

\subsection{Results}

Table 1 provides the peak responses of the structure for the cases of uncontrolled, linear quadratic (LQR) control and proposed Lyapunov based control for all three considered ground motions. It is noted from this table, that the reduction in the peak story displacement without control compared with the LQR control is $36.58 \%, 25.25 \%$ and $13.63 \%$ under El Centro, Northridge and Mexico earthquakes respectively, and the corresponding reductions compared with the proposed Lyapunov control are $69.51 \%, 80.80 \%$ and $80.30 \%$. Reductions in peak acceleration in uncontrolled case compared with the LQR are $31.75 \%, 23.85 \%$ and $11.94 \%$ under El Centro, Northridge and Mexico earthquakes respectively, and the corresponding reductions compared with the proposed Lyapunov control are $54.33 \%$, $36.95 \%$ and $49.84 \%$.

The base shear reduction under El Centro, Northridge and Mexico earthquakes is $36 \%, 25.52 \%$ and $14.35 \%$ respectively compared with the LQR control and $69.44 \%, 81.30 \%$ and 80.38\% compared with Lyapunov control.

From these results, the proposed Lyapunov control is showing better control of responses under all considered ground motions.

Comparisons of evaluation criteria between the LQR case and proposed Lyapunov control are summarized in Table 2. As can be seen from the table, all the corresponding ratios with the proposed Lyapunov control are much smaller than with LQR control under the three records. Reduction ratios are 52.25\%, $33.05 \%, 60.74 \%$ and $38.52 \%$ in displacement, acceleration, RMS displacement and RMS acceleration, respectively under 


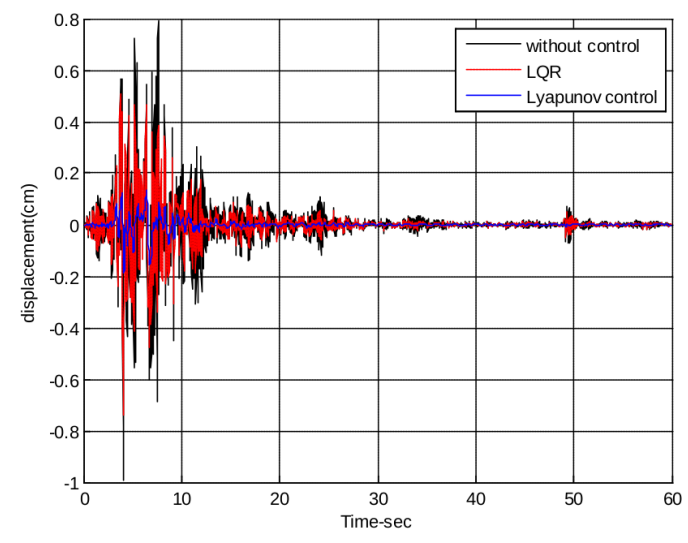

(a) Story displacement

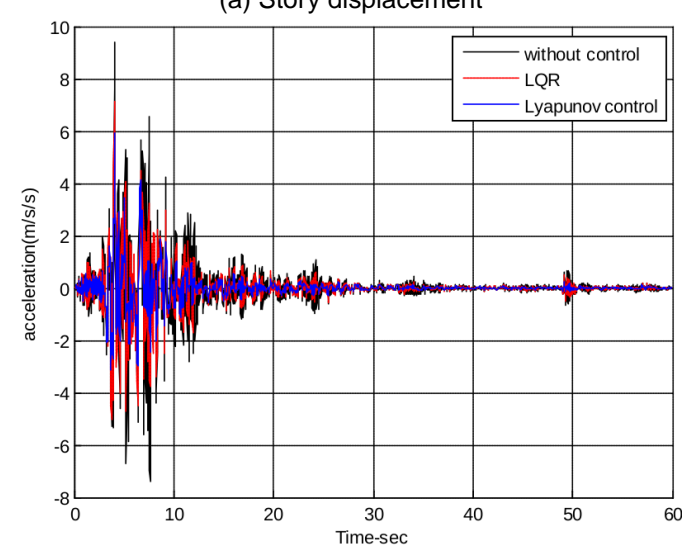

(b) Story acceleration

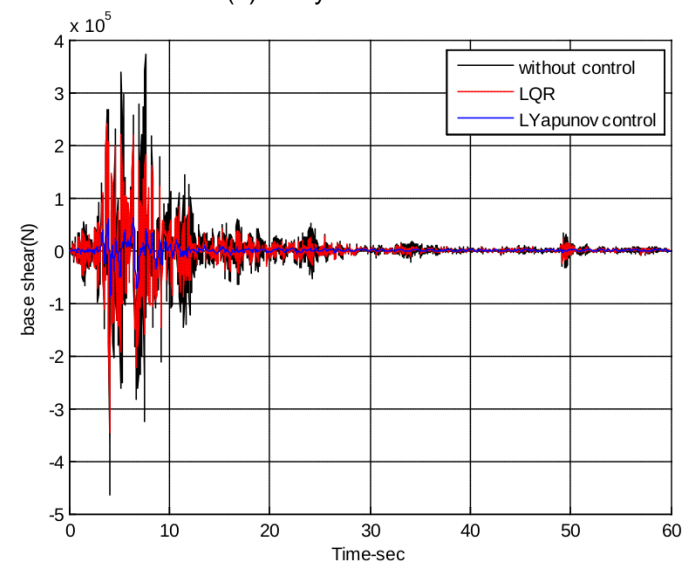

(c) Story base shear

Fig. 3. Uncontrolled and controlled structure responses under Northridge record

El Centro record. The corresponding reduction ratios under Northridge record are $74.89 \%, 17.10 \%, 73.25 \%$ and $29.68 \%$. Under Mexico record, the reduction ratios are $77.09 \%, 43.03 \%$, $63.79 \%$ and $36.33 \%$.

Responses of the structure for the cases of uncontrolled, LQR control and proposed Lyapunov control, in terms of displacement, acceleration and base shear, under the three considered earthquake excitations are depicted in Figures 2, 3 and 4. The figures indicate response reduction for both control algorithms, however it is more significant in case of the proposed Lyapunov control for the three earthquake records.

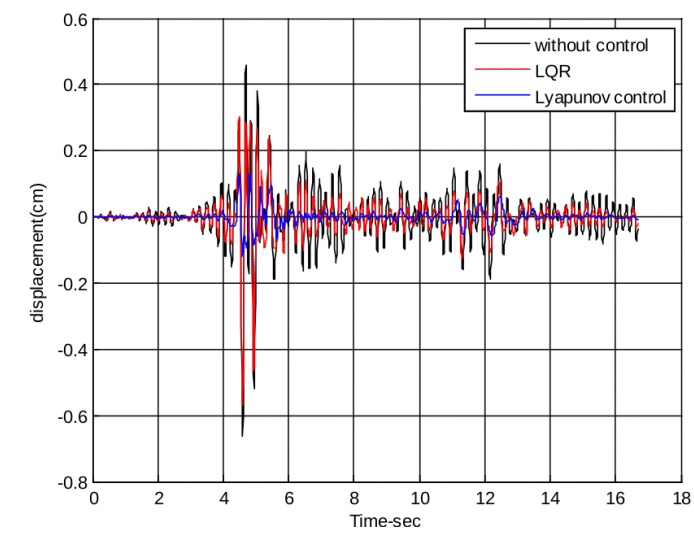

(a) Story displacement

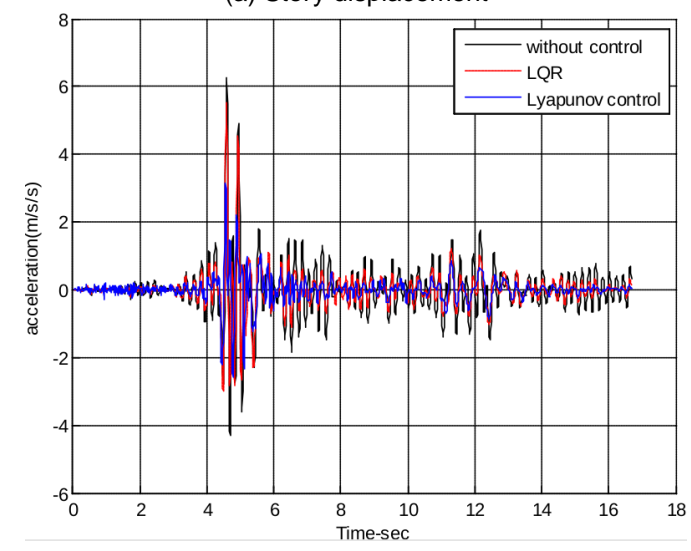

(b) Story acceleration

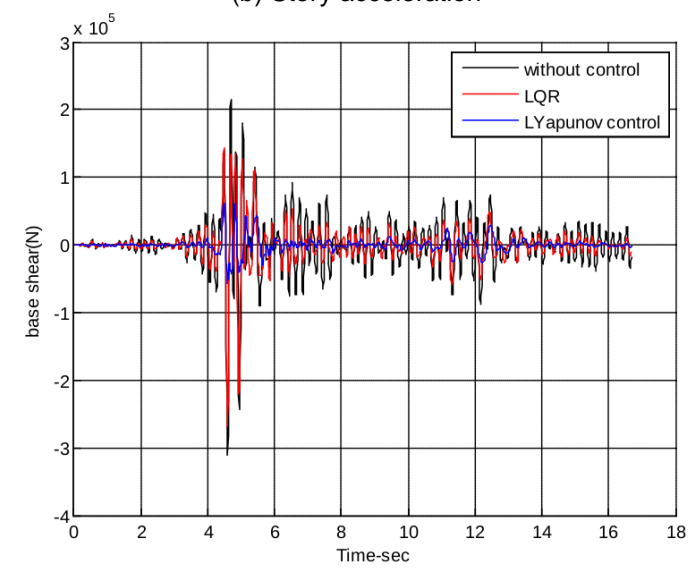

(c) Story base shear

Fig. 4. Uncontrolled and controlled structure responses under Mexico record

\section{Conclusions}

In this study, a new control algorithm based in Lyapunov's stability theory is proposed for SDOF building structures under seismic loading. For its application this proposed control algorithm needs to know the mass, damping and stiffness of the structure. A quadratic Lyapunov function has been considered to develop the control algorithm. The performance of this algorithm is demonstrated by numerical simulations on a single story shear building structure subjected to three historical ground motions, El Centro 1940, Northridge 1994 and Mexico 1978 which still used in research studies and practical control applications. Simulation results and the evaluation criteria show the effectiveness of the proposed Lyapunov controller in reducing displace- 
ment and acceleration considerably. Further reduction in base shear is also obtained. Another advantage of this method is that the stability of the structure can be verified without solving the dynamic equation explicitly.

As future work, the proposed Lyapunov based control will be extend to two and multiple story building (MDOF).

\section{References}

1 Nishtani A, Inoue Y, Overview of the application of active/semiactive control to building structures in Japan, Earthquake Engineering and Structural Dynamics, 30(11), (2001), 1565-1574, DOI 10.1002/eqe.81

2 Datta T K, A state-of-the-art review on active control of structures, ISET Journal of Earthquake Technology, 40(1), (2003), 1-17.

3 Ha Q P, Kwok N M, Nguyen M T, Li J, Samali B, Mitigation of seismic responses on building structures using MR dampers with Lyapunov-based control, Structural Control and Health Monitoring, 15(4), (2008), 604-621, DOI $10.1002 /$ stc. 218

4 Jansen L M, Dyke S J, Semiactive control strategies for MR dampers: comparative study, Journal of Engineering Mechanics, 126(8), (2000), 795-803, DOI 10.1061/(ASCE)0733-9399(2000)126:8(795)

5 Aldemir Ü, Bakioglu M, Semi active control of earthquake-excited structures, Turkish Journal of Engineering and Environmental Sciences, 24, (2000), 237-246.

6 Arfiadi Y, Hadi M N S, Continuous bounded controllers for active control of structures, Computer and Structures, 84(12), (2006), 798-807, DOI 10.1016/j.compstruc.2006.01.001

7 Lee S H, Min K W, Lee Y C, Modified sliding mode control using a target derivative of the Lyapunov function, Engineering Structures, 27(1), (2005), 49-59, DOI 10.1016/j.engstruct.2004.08.010

8 Choi KM, Lee H-J, Cho S-W, Lee I W, Modified energy dissipation algorithm for seismic structures using Magnetorheological damper, KSCE Journal of Civil Engineering, 11(2), (2007), 121-126, DOI 10.1007/BF02823855

9 Zaccarian L, Nesic D, Teel A R, Analytical and numerical Lyapunov functions for SISO linear control systems with first order reset elements, International Journal of Robust and Nonlinear control, 21(10), (2011), 1134-1158, DOI $10.1002 /$ rnc.1649

10 Udwadia F E, A new approach to stable optimal control of complex nonlinear dynamical systems, Journal of Applied Mechanics, 81(31), (2014), 1-6, DOI $10.1115 / 1.4024874$

11 Wu Z, Soong T T, Modified bang-bang control law for structural control implementation, Journal of Engineering Mechanics ASCE, 122(8), (1996), 71-777, DOI 10.1061/(ASCE)0733-9399(1996)122:8(771)

12 Min K W, Hwang J S, Lee S H, Chung L, Probabilistic approach for active control based on structural energy, Earthquake Engineering and Structural dynamics, 32(6), (2003), 2301-2318, DOI 10.1002/eqe.327

13 Hiramoto K, Matsuoka T, Sunakoda K, Inverse Lyapunov approach for semi-active control of civil structures, Structural Control and Health Monitoring, 18, (2011), 382-403, DOI 10.1002/stc.375

14 MATLAB, the language of technical computing, the Mathworks Inc.

15 Djajakesukma SL, Samali B, Nguyen H, Study of A semi-active stiffness damper under various earthquake inputs, Earthquake Engineering and Structural dynamics, 31, (2002), 1757-1776, DOI 10.1002/eqe.181

16 Ohtori Y, Christenson RE, Spencer Jr Bf, Dyke SJ, Benchmark control problems for Seismically excited nonlinear buildings, Journal of Engineering Mechanics, 130(4), (2004), 366-385, DOI 10.1061/(ASCE)07339399(2004)130:4(366) 